\title{
SPIN EVOLUTION OF THE PROGENITORS OF BINARY AND MILLISECOND PULSARS
}

\author{
PRANAB GHOSH \\ Tata Institute of Fundamental Research \\ Bombay 400 005, INDIA
}

\section{Introduction}

In this symposium, I have been given the task of summarizing our current understanding of the evolutionary history of spin periods of the neutron stars that we now see as binary and millisecond pulsars, i.e., recycled pulsars. We believe that a newborn, fast-spinning neutron star (with a rather high magnetic field $\sim 10^{11}-10^{13} \mathrm{G}$ ) in a binary system first operates as a spin-powered pulsar, subsequently as an accretion-powered pulsar when accretion begins after the pulsar has been spun down adequately, and finally as a spin-powered pulsar for the second time after having been recycled to become a very fast-rotating neutron star (with a rather low magnetic field $\sim 10^{8}-10^{11} \mathrm{G}$ ) (see Ghosh 1994a,b, hereafter G94a,b).

A magnetic field-spin period $(B-P)$ diagram of neutron stars, as shown in Fig. 1, is widely used for studying spin evolution (indeed, Dr. Bailes has called it the Hertzsprung-Russell diagram of pulsars!). In addition to the "pulsar island", Fig. 1 shows the recycled pulsars (i.e., those in the second spin-powered phase) at the bottom left of the diagram. Recycled pulsars can also be single, of course, since the binary can be disrupted (by processes that are mentioned in Section 4) before the second spin-powered phase begins. The binary X-ray pulsars (i.e., those in the accretion-powered phase) are at the top right of Fig. 1: only those $10 \mathrm{X}$-ray pulsars for which direct measurements of the magnetic field from a cyclotron line are currently available are shown. Of these, 9 were observed with GINGA (Nagase 1992), and the cyclotron feature in A $0535+26$ has been recently observed with OSSE (Grove et al. 1994). Fig. 1 also shows the two currently known binary neutron stars which are thought to be in their first spin-powered phase, namely, PSR B1259-63 and PSR J0045-73 (Johnston et al. 1994, Johnston 


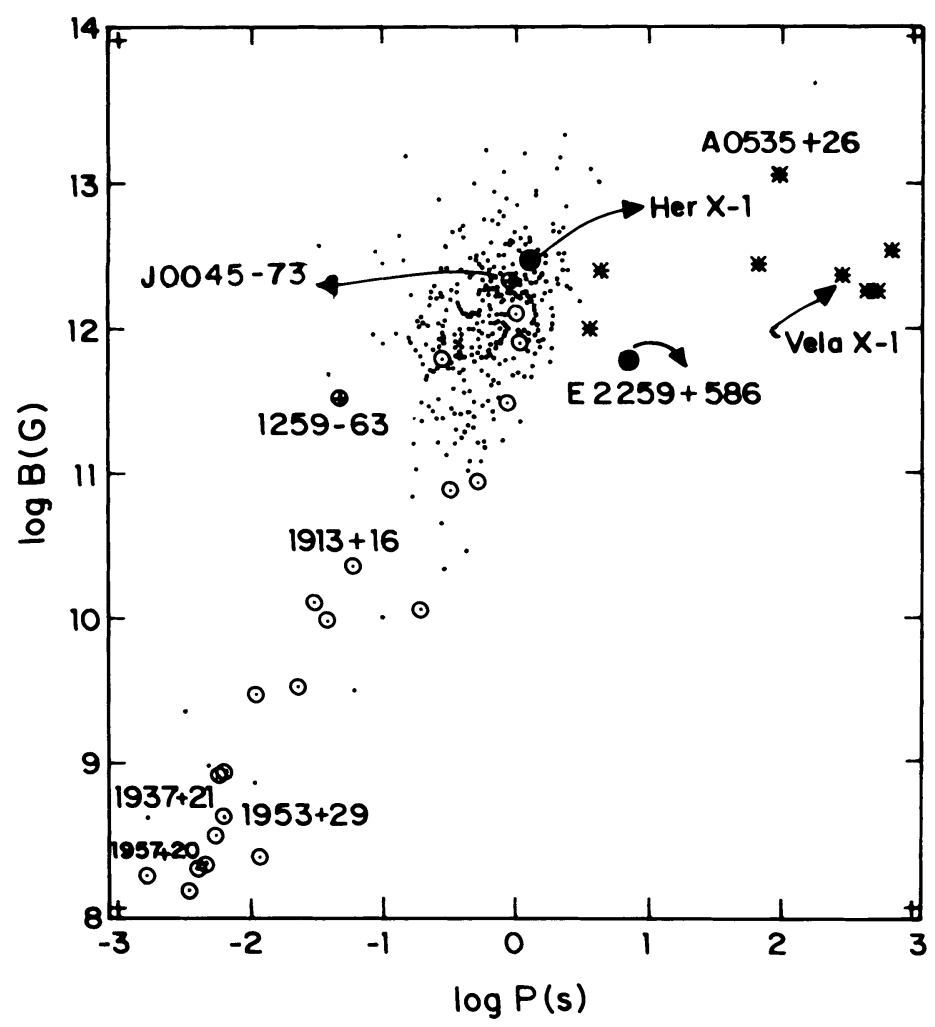

Figure 1. Spin- and accretion-powered pulsars on the $B-P$ diagram. Dots indicate spin-powered pulsars, and asterisks, accretion-powered ones. Spin-powered pulsars in binaries are encircled, and the two recently discovered massive radio pulsar binaries (PSRs B1259-63 and J0045-73) have an additional cross on their symbols. Some well-known pulsars are marked individually: these are (a) several recycled pulsars, (b) PSRs B1259-63 and J0045-73, and, (c) three accretion-powered pulsars, two of them with relatively low-mass companions (Her X-1 and E 2259+586), and one with a massive companion (Vela X-1).

1994, hereafter J94a,b; Kaspi et al. 1994): we have heard about these two massive radio binary pulsars in detail from Drs. Johnston and Kaspi in this symposium.

\section{Initial Spin Down}

During the initial spin down the neutron star moves rightward on the $B-P$ diagram along an approximately horizontal track. 


\subsection{BRAKING TORQUES}

The electromagnetic braking torque, which dominates the spin down at the fastest spin-rates, scales as $N \propto \mu^{2}\left(\Omega_{\mathrm{s}} / c\right)^{3}$, where $\mu$ is the magnetic dipole moment of the neutron star, and $\Omega_{\mathrm{s}}$ is its angular velocity. As the star spins down, plasma torques begin to dominate over the electromagnetic torque. This can be readily seen by comparing the electromagnetic torque with the so-called subsonic propeller torque, $N \propto \mu^{2} \Omega_{\mathrm{s}}^{2} / G M$ (Illarionov \& Sunyaev 1975, hereafter IS; Mineshige et al. 1991; see Henrichs 1983 for a review). The latter torque obviously dominates for $P>P_{\text {crit }} \propto G M / c^{3} \sim 2 \mathrm{~ms}$. Actually, the subsonic propeller torque is an upper limit to the possible plasma torques. In reality, the plasma torque relevant at the highest spin rates is the so-called supersonic propeller torque, which is smaller by a factor $\sim\left(c_{\mathrm{s}} / \Omega_{\mathrm{s}} r_{\mathrm{A}}\right)$ than the subsonic torque, so that $P_{\text {crit }} \sim 50-100 \mathrm{~ms}$ for typical mass-flow rates from a massive companion to the neutron star. Here, $c_{\mathrm{s}}$ is the sound speed, and $r_{\mathrm{A}}$ is the Alfvén radius. For $c_{\mathrm{s}} \sim v_{\mathrm{ff}}$ at $r_{\mathrm{A}}$, the supersonic propeller torque reduces to the original IS scaling, i.e., $N \propto\left(\mu^{2} / r_{\mathrm{A}}^{3}\right)\left(\Omega_{\mathrm{K}}\left(r_{\mathrm{A}}\right) / \Omega_{\mathrm{s}}\right)$. Here, $v_{\mathrm{ff}}$ is the free-fall velocity.

\subsection{SPIN DOWN OF PSR B1259-63}

Studies of the spin down history of PSR B1259-63, a neutron star-Be star (SS 2883) binary with a highly eccentric $(e \simeq 0.87) 3.4 \mathrm{yr}$ orbit (J94a,b), are now providing us with the first detailed look at the processes of initial spin down. Far away from the periastron, the $47 \mathrm{~ms}$ pulsar undergoes spin down with a characteristic age of $3.310^{5} \mathrm{yr}$, predominantly by electromagnetic torques, which implies a magnetic field $\sim 3.310^{11} \mathrm{G}$ for the neutron star (J94a). Close to the periastron, propeller-type torques due to the dense plasma in the equatorial disk around the Be star, as well as those due to the more tenuous plasma in the stellar wind, contribute appreciably to spin down, and may well dominate the instantaneous spin down rate $($ G94a,b) as the pulsar passes through the disk. The situation is shown in Fig. 2, which displays the spin down rates $(\dot{P})$ due to the above torques in a polar diagram: to read off $\dot{P}$ due to a particular torque at a given binary phase, one simply draws a line from the origin (the position of SS 2883) towards the position of the pulsar at that phase (the binary orbit is shown in Fig. 2), until the line meets the curve for that particular torque. $\dot{P}$ is just the length of this line. It is clear from Fig. 2 that the values of $\dot{P}$ for the electromagnetic torque and for the propeller torque in the (tenuous) stellar wind are generally comparable, while that for the propeller torque in the (dense) disk of SS 2883 is considerably higher (indeed, parts of the relevant $\dot{P}$ curve are out of scale in Fig. 2).

For the model computations (Ghosh 1994c, hereafter G94c) shown in 


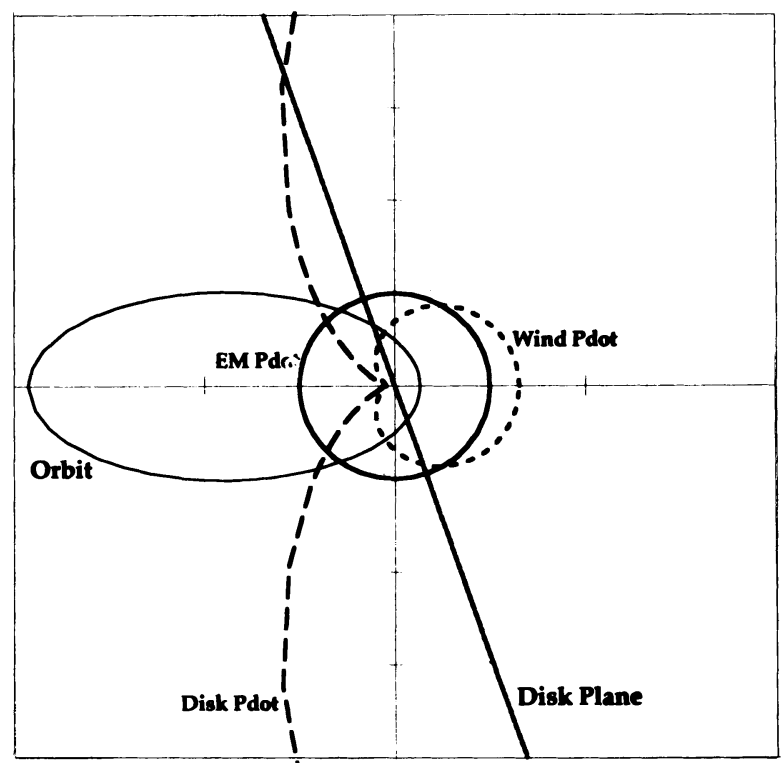

Figure 2. Spin down of PSR B1259-63. Shown as polar plots are the spin down rates for the electromagnetic torque, and the plasma torques due to the disk and the wind of SS 2883 (see text). Also shown are the binary orbit and the line of intersection of the disk plane with the orbital plane. Note that SS 2883 is at the origin, and the angle between the $y$-axis and the disk plane $\left(\lesssim 10^{\circ}\right)$ is shown exaggerated for clarity.

Fig. 2, I have used the disk- and wind-density profiles $(\rho)$ given by both the theoretical calculations of Bjorkman \& Cassinelli (1993, hereafter BC) and by the model-fitting of Be-star observations by Waters (1986, hereafter $\mathrm{W})$, i.e., profiles of the type $\rho=\rho_{0}\left(r / R_{*}\right)^{-n}$, where $R_{*}$ is the stellar radius. The density scale, $\rho_{0}$ is set by the requirements that (a) the density of the disk where it touches the stellar surface is $\sim 10^{-11}-10^{-12} \mathrm{~g} \mathrm{~cm}^{-3}$ and (b) the wind density at the stellar surface is $\sim 10^{-2}-10^{-3}$ of the disk density. These numbers are in accordance with both $\mathrm{BC}$ and $\mathrm{W}$. The exponent $n$ is $\simeq 2$ for the wind, and $\simeq 3$ to 4 for the disk, again in accordance with both $\mathrm{BC}$ and $\mathrm{W}$. Further, the propeller torque relevant here is the supersonic one in the IS formulation (see above), which yields for the wind spin down rate $(\mathrm{G} 94 \mathrm{c}) \dot{P}_{\text {wind }} / \dot{P}_{\mathrm{EM}} \simeq 0.7(1+e \cos \theta)$ and the disk spin down rate $\dot{P}_{\text {disk }} / \dot{P}_{\mathrm{EM}} \simeq 7(1+e \cos \theta)^{2}$ relative to the electromagnetic spin down rate $\dot{P}_{\mathrm{EM}}$. Here, $e$ is the orbital eccentricity, $\theta$ is the true anomaly, and I have taken $n \simeq 4$ for the disk (W, J94b).

While $\dot{P}_{\text {disk }}$ is quite large near periastron, the amount of additional spin down produced by this mechanism during periastron passage (which is the observed quantity) depends crucially on the orientation of the disk plane relative to the orbital plane $(\mathrm{G} 94 \mathrm{~b}, \mathrm{c})$. The two planes tend to become aligned in older systems due to tidal torques. However, this need not 
be the case in a young system like PSR B1259-63/SS 2883, and there are indications already that it is not. There is little perturbation to the $\mathrm{H} \alpha$ emission from the system during periastron passage (Manchester, private communication). This emission is thought to originate in the disk, which would be grossly perturbed by the tidal torques near periastron if the two planes were coaligned (Kochanek 1993), while an appreciable misalignment $\left(~\left(30^{\circ}\right.\right.$, say) would be consistent with observation. This picture is also consistent with the dates (J94b) on which the pulsar disappeared ( $\sim$ December $20,1993)$ before and reappeared ( $\sim$ February 2,1994$)$ after the periastron passage of January 9,1994 , since these dates imply an angular separation $\sim 200^{\circ}$ between these two points, as would be expected for a misaligned disk with an opening angle $\sim 15^{\circ}$ (as advocated by W) intersecting the orbit near these points (G94c). For this disk configuration, $\Delta P$ is increased by $\sim 50 \%$ over the amount expected from the electromagnetic torque (G94c) by the two passages (see Fig. 2) through the disk; this number is consistent with the preliminary results from the peristron observations described above (Johnston, private communication). A much thinner disk (of opening angle $\sim 1^{\circ}$ ), as advocated by $\mathrm{BC}$, would give an enhancement which is $\sim 10$ times as small as the above value, while an aligned disk would give an enhancement that is $\sim 10$ times as big (G94c).

\section{Spin Evolution in the Accretion-Powered Phase}

Since this is not the main focus of my talk, only the essential points are summarized here; for more details, see G94a,b. Under the action of accretion torques, the pulsar can undergo both spin up and spin down, so that it moves back and forth on approximately horizontal tracks in the region occupied by accretion-powered pulsars in Fig. 1. A crucial feature of the accretion torque is that it changes sign at a critical value of the pulsar's spin rate: the corresponding critical value, $\omega_{\mathrm{c}}$, of the dimensionless fastness parameter $(\mathrm{G} 94 \mathrm{a}, \mathrm{b})$ is now becoming constrained by recent studies of the detailed period histories of binary X-ray pulsars: current indications are that $\omega_{\mathrm{c}} \sim 0.4-0.7$.

\section{Final Spin Up}

During this process, the neutron star moves downward and left in Fig. 1, from the area occupied by accretion-powered pulsars to the (roughly) linear band occupied by recycled pulsars; the upper edge of this band being the socalled spin up line (see Bhattacharya \& Van den Heuvel 1991). The essential process at work here is the accretion torque: indeed, the explanation of the periods of the recycled pulsars was a major triumph of the accretion torque theory in the 1980's. 


\subsection{SPIN EVOLUTION}

The final evolution of high-mass X-ray binaries (HMXBs) can go in two ways when the common-envelope (CE) phase begins (Van den Heuvel 1992 and references therein, hereafter vdH92). An initially wide binary (e.g., a Be star system) can eject the entire envelope and produce a neutron star with a helium core companion. The system then evolves either (a) by a supernova explosion of the companion, or, (b) by evolution of the companion into a massive white dwarf. In the former case, the chances are high that the system remains bound, producing a double neutron star system like PSR 1913+16; if it does become unbound, two runaway pulsars are produced, one of which is recycled. In the latter case, a system like PSR 0655+64 is thought to be produced. The spin evolution is qualitatively straightforward in both cases: the neutron star is spun up to short periods ( $\sim 50-1000 \mathrm{~ms}$, say) determined by the strength of the full-scale Roche lobe overflow (that initiates the CE phase) and the magnetic field of the neutron star. On the other hand, an initially narrow binary undergoes a complete spiral-in in the CE phase, producing a Thorne-Zytkow object (vdH92) with a disk-accreting neutron star in its core. The end product is a recycled, spun up, single radio pulsar.

Neutron stars with low magnetic fields $\left(\sim 10^{8}-10^{9} \mathrm{G}\right)$ in very bright (near-Eddington) low-mass X-ray binaries (LMXBs) are thought to be spun up to $\sim$ millisecond periods by accretion torques, and these systems are then believed to evolve into binary pulsars of the PSR $1953+29$ class (vdH92, Webbink 92).

\subsection{ACCRETION-INDUCED FIELD DECAY}

It is clear from Fig. 1 that magnetic fields of neutron stars are reduced considerably during the passage from the accretion-powered phase to the second spin-powered phase. A connection between this magnetic-field decay and the accretion during the final spin up phase is obviously possible, and this has been a subject of much study recently (see Verbunt 1994). The magnetic-field distribution of recycled pulsars appears bimodal (see Kulkarni 1992; G94b): the low-magnetic-field pulsars are those which descended from LMXBs (i.e., the "1953+29 Class", or LMBPs, see vdH92) with relatively large amounts of mass accreted during the long-lived final spin up phase, and the high-magnetic-field pulsars are those which descended from HMXBs (i.e., the "1913+16 Class", vdH92) with relatively little mass accreted during the short-lived final spin up phase (G94b).

A valuable diagnostic of accretion-induced field decay comes from the correlation between the magnetic fields and orbital periods of LMBPs, first noticed by Van den Heuvel (1994; see also De Kool \& Van Paradijs 1987), 


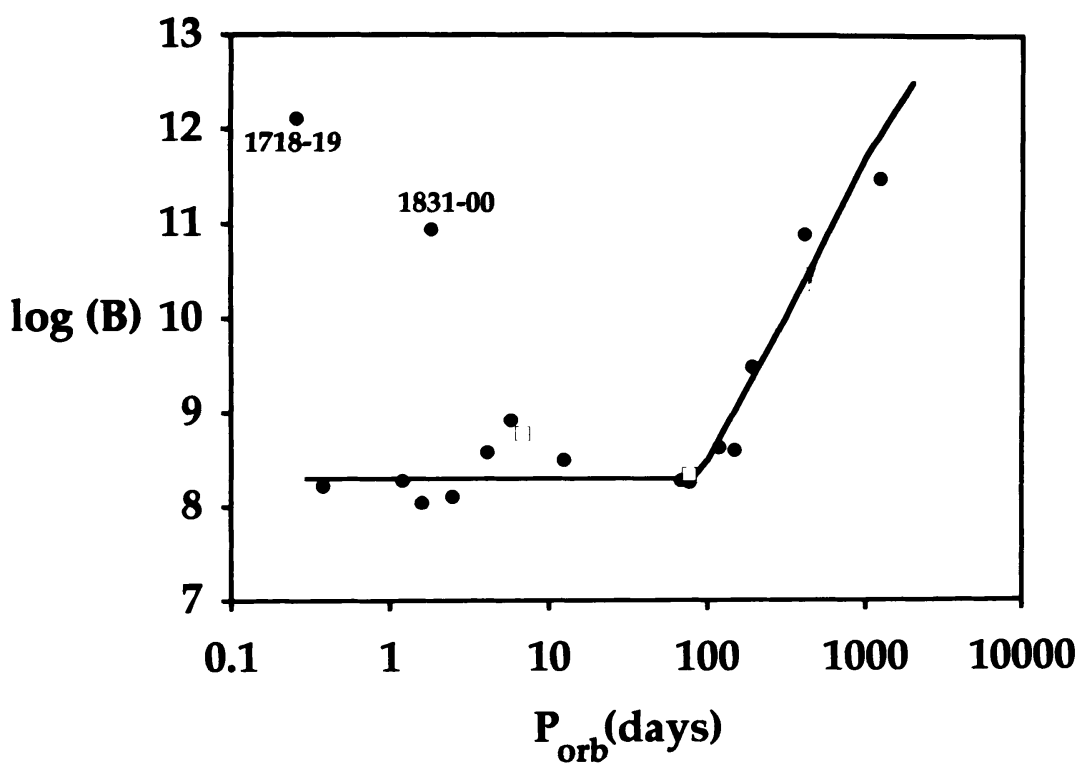

Figure 3. Magnetic field-orbital period correlation for low-mass binary pulsars (LMBPs), after Van den Heuvel (1994). All the $18 \mathrm{LMBPs}$ with measured $B$ as of August 1994 have been included. Squares denote pulsars with only an upper limit on $B$.

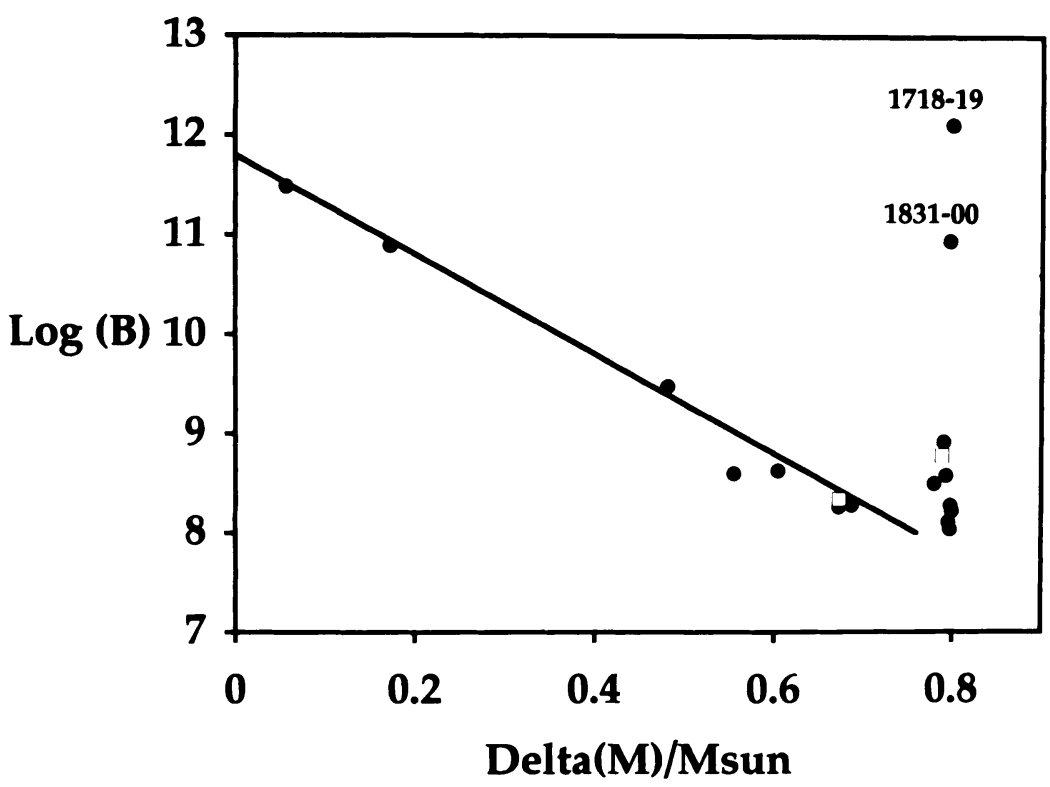

Figure 4. Accretion induced field decay. Shown are the $18 \mathrm{LMBPs}$ from Fig.3. 
and shown in Fig. 3. It is well known from the evolutionary calculations (Joss et al. 1987; Pylyser \& Savonije 1988) of such binaries that there is a one-to-one relation between the mass transferred $(\Delta M)$ during the LMXB evolution and the final orbital period of the system. Using this relation, I get the remarkable description for accretion induced field decay shown in Fig. 4: the relation between $B$ and $\Delta M$ can be approximated by $\log B \simeq 11.8-\left(\Delta M / 0.2 M_{\odot}\right)$.

Does Fig. 4 really imply an exponential field deacy, which runs contrary to the prevalent folklore of power-law field decay? On the one hand, we must be careful in interpreting $\Delta M$ in Fig. $4:$ it is the mass lost by the companion, which need not all be accreted. On the other, model calculations of neutron-star conductivity often give $(B-\Delta M)$ relations which are not really power-laws but mimic them over a restricted range. In any case, it is clear that this diagnostic has important implications both for the position of the spin up line (Ghosh \& Verbunt, in preparation) and for models of neutron-star conductivity (Ghosh \& Pethick, in preparation).

\section{References}

Bhattacharya, D. \& Van den Heuvel, E.P.J. 1991, Phys. Rep. 203, 1

Bjorkman, J.E. \& Cassinelli, J.P. 1993, ApJ 409, 429 (BC)

De Kool, M. \& Van Paradijs, J. 1987, A\&A 173, 279

Ghosh, P. 1994a, in The Evolution of X-ray Binaries, S.S. Holt \& C.S. Day (Eds.), AIP Conf. Proc. Vol. 308, p. 439 (G94a)

Ghosh, P. 1994b, in Pulsars: Festschrift for V. Radhakrishnan, G. Srinivasan (Ed.), Indian Academy of Sciences (Bangalore), (in press) (G94b)

Ghosh, P. 1994c, ApJ (submitted) (G94c)

Grove, E. et al. 1994, ApJ (submitted)

Henrichs, H. 1983, in Accretion-Driven Stellar X-ray Sources, W.H.G. Lewin \& E.P.J. van den Heuvel (Eds.), Cambridge Univ. Press, p. 393

Illarionov, A.F. \& Sunyaev, R.A. 1975, A\&A 39, 185 (IS)

Johnston, S. et al. 1994a, MNRAS 268, 430 (J94a)

Johnston, S. 1994b, these Proceedings (J94b)

Joss, P.C., Rappaport, S. \& Lewis, W. 1987, ApJ 319, 180

Kaspi, V.M. et al. 1994, ApJ 423, L43

Kochanek, C. 1993, ApJ 406, 638

Kulkarni, S.R. 1992, Phil. Trans. Roy. Soc. London A341, 77

Mineshige, S., Rees, M.J. \& Fabian, A.C. 1991, MNRAS 251, 555

Nagase, F. 1992, in Proc. Ginga Memorial Symposium, F. Makino \& F. Nagase (Eds.), ISAS, p. 1

Pylyser, E. \& Savonije, G.J 1988, A\&A 191, 57

Van den Heuvel, E.P.J. 1992, in $X$-ray Binaries $\&$ Recycled Pulsars, E.P.J. van den Heuvel \& S. Rappaport (Eds.), Kluwer Academic Publishers, p. 233 (vdH92)

Van den Heuvel, E.P.J. 1994, A\&A (submitted)

Verbunt, F. 1994, in The Evolution of X-ray Binaries, S.S. Holt \& C.S. Day (Eds.), AIP Conf. Proc. Vol. 308, p. 351

Waters, L.B.F.M. 1986, A\&A 162, 121 (W)

Webbink, R.F. 1992, in X-ray Binaries \& Recycled Pulsars, E.P.J. van den Heuvel \& S. Rappaport (Eds.), Kluwer Academic Publishers, p. 269 